\title{
Effect Evaluation of Cognitive Behavioral Intervention on Stress Response in Breast Cancer Patients
}

\author{
Ping Huang1, You Li1 ${ }^{1}$, Linqiu Fu², Xiangling Jiang2*, Juncheng Guo ${ }^{3}$, Yijun Yang3, Chunru Wang4, \\ Yunwang $\mathrm{Cai}^{2}$, Min Guo² \\ ${ }^{1}$ University of South China, Hengyang, China \\ ${ }^{2}$ Hainan Provincial People's Hospital, Haikou, China \\ ${ }^{3}$ Haikou Hospital Affiliated to Xiangya School of Medicine, Central South University, Changsha, China \\ ${ }^{4}$ Qionghai People’s Hospital, Qionghai, China \\ Email: *g2002m@163.com
}

How to cite this paper: Huang, P., Li, Y., Fu, L.Q., Jiang, X.L., Guo, J.C., Yang, Y.J., Wang, C.R., Cai, Y.W. and Guo, M. (2019) Effect Evaluation of Cognitive Behavioral Intervention on Stress Response in Breast Cancer Patients. Journal of Behavioral and Brain Science, 9, 121-129.

https://doi.org/10.4236/jbbs.2019.93010

Received: February 25, 2019

Accepted: March 18, 2019

Published: March 21, 2019

Copyright $\odot 2019$ by author(s) and Scientific Research Publishing Inc. This work is licensed under the Creative Commons Attribution International License (CC BY 4.0).

http://creativecommons.org/licenses/by/4.0/

\begin{abstract}
Objective: To investigate the effects of cognitive behavioral intervention on psychological stress response in breast cancer patients by cognitive behavioral intervention in breast cancer patients. Methods: Adopting the random comparison method, 100 patients with breast cancer were divided into comparison group and intervention group by 50 cases. The intervention group underwent cognitive behavioral intervention at the same time as the comparison group only received conventional treatment without cognitive behavioral intervention. Two groups of breast cancer patients were enrolled in the general questionnaire, the Self-Rating Anxiety Scale (SAS), and the Medical Coping Modes Questionnaire (MCMQ) within one week after admission and one month after treatment to understand the psychological stress levels of the two groups. Results: There was no significant difference in the scores of SAS and MCMQ between the intervention group and the comparison group before intervention $(\mathrm{P}>0.05)$. But after intervention, the scores of SAS and MCMQ in the comparison group were significantly higher than those in the intervention group $(\mathrm{P}<0.05)$; thus, the differences of SAS and MCMQ factors before and after intervention in the intervention group were statistically significant $(\mathrm{P}<0.05)$, and there was no significant difference in the factors of SAS and MCMQ before and after intervention in the comparison group $(P>0.05)$. It shows that the implementation of cognitive behavioral intervention therapy for breast cancer patients has the effect of improving their psychological status. Conclusion: Cognitive behavioral intervention therapy combined with psychology for breast cancer patients can effectively reduce their stress level, improve mental health status and improve their positive coping ability.
\end{abstract}




\section{Keywords}

Cognitive Behavioral Therapy, Breast Cancer, Stress Response, Coping Style

\section{Introduction}

In recent years, with the changes in the social environment, the increasing pressure on people's lives and the unhealthy lifestyles result that the incidence of cancer in the world has shown an overall trend of increasing year by year, which seriously endangers the physical and mental health of human beings. In particular, breast cancer has become one of the most common malignant tumors in the world, accounting for about $18 \%$ of female cancers. In the western developed countries, breast cancer has become the second most common malignant tumor in women other than skin cancer. Although China is currently a low-incidence area for breast cancer, in recent years, the number of new cases of breast cancer has increased by $3 \%$ to $5 \%$ annually, which is significantly higher than the annual average growth rate of the world; China has become the fastest growing country for breast cancer [1]. Especially in economically developed areas and large cities such as Beijing, Shanghai, Guangzhou, etc., the incidence of breast cancer has been the highest and second in the incidence of female malignant tumors [2]. Breast cancer has gradually become the number one killer threatening the health of modern Chinese women. Although with the continuous improvement of modern medical technology and continuous improvement of surgical methods, the life cycle and survivors of breast cancer patients have been prolonged and increased year by year, the double pain of cancer itself to the physical and psychological aspects of patients is still inexplicable. And because of the specificity of the breast for the shape of women, the severity of the psychological stress response of patients is more prominent than other cancer patients. Because breast cancer patients in the course of treatment, not only have to endure the body pain caused by the disease and a series of side effects caused by chemotherapy, but also suffer from the huge psychological pain such as breast loss and physical damage. These psychological and physiological factors can lead to severe psychological stress in breast cancer patients. Stress is an environment-dependent stress response state caused by the imbalance of physiological and psychological objective needs and coping ability of organisms under certain environmental stimuli [3]. When the stressor is gentle and the action time is too short, it is a defense reaction that the body presents to adapt to changes in the internal and external environment, and is a self-protective reaction of the human body. It is conducive to the movement of the body's potential, preparation status and confrontation. However, once the stress continues to rise and its effect is too strong, high catabolism will cause a negative nitrogen balance, and the patient will experience symptoms such as weakness and even death, which will have serious adverse effects on the body, seriously affecting and disturbing the patient's 
mentality.

Surgery is one of the main treatments for breast cancer patients, most breast cancer patients will become more and more psychologically stressed as the surgery day gets closer. Combined with the physiological factors such as medicine and pain, they will make the patient a huge physiological and psychological stress response, resulting in a series of physiological disturbances, negative emotions and negative coping styles, which greatly reduces the effect of surgical treatment, affects the postoperative rehabilitation and quality of life of patients. A large number of studies [4] [5] [6] [7] have shown that the quality of life of cancer patients are closely related to their psychological factors and coping styles. Effective psychological intervention therapy for breast cancer patients can alleviate the patient's stress response to a certain extent, improve the patient's ability to cope, and thus improve the quality of life of patients.

\section{Study Object and Method}

\subsection{Study Object}

The study included 100 breast cancer patients who met the inclusion criteria for the first breast cancer modified radical mastectomy in Hainan Provincial People's Hospital from 2016 to 2017. They were randomly divided into 50 groups in the comparison group and intervention group respectively. Inclusion criteria: 1) Early and mid-stage breast cancer patients by pathological and B-ultrasound diagnosis; 2) Patients older than 18 years of age; 3) No other serious systemic disease history or mental illness; 4) With basic reading and writing ability, can complete the questionnaire independently and volunteer to participate in the study. Exclusion criteria: 1) Patients with advanced breast cancer; 2) Patients older than 65 years of age; 3) People with mental illness; those with severe heart, liver, kidney and other dysfunctions or other malignant tumors; 4) Without basic reading and writing ability, cannot complete the questionnaire independently or refuse to participate in the study. The patients in the intervention group were $53.16 \pm 9.34$ years old and weighed $62.54 \pm 10.12 \mathrm{~kg}$. The education level was 14 cases in junior high school and below, 19 cases in high school (technical secondary school), and 27 cases in junior college and above. The average age of the comparison group was $54.16 \pm 8.69$ years old, and weighed 64.11 $\pm 12.03 \mathrm{~kg}$. The education level was 12 cases in junior high school and below, 20 cases in high school (technical secondary school), and 18 cases in junior college and above. There was no significant difference in age and weight between the two groups $(P>0.05)$. There was no statistically significant difference between the two groups in terms of education level, occupation, medical expenses, and other demographic data $(P>0.05)$. See Table 1 for details.

\subsection{Study Tools}

\subsubsection{Self-Rating Anxiety Scale (SAS)}

The Self-Rating Anxiety Scale (SAS) is used to reflect the subjective feelings of 
Table 1. Comparison of general data between the two groups of patients.

\begin{tabular}{|c|c|c|c|c|c|}
\hline Item & Class & $\begin{array}{c}\text { Intervention } \\
\text { Group }(\mathrm{n}=50)\end{array}$ & $\begin{array}{c}\text { Comparison } \\
\text { Group }(\mathrm{n}=50)\end{array}$ & Statistics & P Value \\
\hline Age & & $53.27 \pm 9.34$ & $54.16 \pm 8.69$ & $t=0.493$ & 0.311 \\
\hline Weight & & $62.54 \pm 10.12$ & $64.11 \pm 12.03$ & $t=0.706$ & 0.241 \\
\hline \multirow{3}{*}{$\begin{array}{c}\text { Educational } \\
\text { level }\end{array}$} & $\begin{array}{c}\text { Junior high } \\
\text { school and below }\end{array}$ & 14 & 12 & & \\
\hline & $\begin{array}{c}\text { High school } \\
\text { (secondary school) }\end{array}$ & 19 & 20 & $Z=0.206$ & 0.902 \\
\hline & College and above & 17 & 18 & & \\
\hline \multirow{5}{*}{ Occupation } & Institution & 4 & 6 & & \\
\hline & Business unit & 13 & 12 & & \\
\hline & Self-employed & 18 & 17 & $x^{2}=1.069$ & 0.899 \\
\hline & Farmer & 11 & 9 & & \\
\hline & other & 4 & 6 & & \\
\hline \multirow{3}{*}{$\begin{array}{l}\text { Monthly per } \\
\text { capita income }\end{array}$} & Less than 3000 yuan & 23 & 21 & & \\
\hline & $3000-6000$ & 19 & 22 & $Z=0.373$ & 0.829 \\
\hline & More than 6000 yuan & 8 & 7 & & \\
\hline \multirow{5}{*}{$\begin{array}{l}\text { Medical } \\
\text { expenses }\end{array}$} & Medical insurance & 22 & 21 & & \\
\hline & $\begin{array}{l}\text { Rural cooperative } \\
\text { medical care }\end{array}$ & 25 & 27 & $x^{2}=0.301$ & 0.861 \\
\hline & Self-pay & 3 & 2 & & \\
\hline & Ethnic group Han & 44 & 46 & $x^{2}=0.445$ & 0.506 \\
\hline & Other & 6 & 4 & & \\
\hline
\end{tabular}

Note: $\mathrm{P}>0.05$ was not statistically significant.

patients with anxiety symptoms, and to measure the severity of their anxiety and the self-assessment psychological scale of patients with changes during treatment. This scale contains 20 items, using a four-level scoring standard, and the total scores are the sum of all the items. 5 items in the entire scale marked with the ${ }^{\star}$ number is for the reverse score, and the scoring standard is reversed from the back to the front. The result of the evaluation is the sum of the scores of all the items and multiplied by 1.25 , taking the integer part, which is then the standard score. The cut-off value of the standard score of the normative results of the anxiety self-assessment table is 50 points. When the patient's standard score exceeds the cut-off value, we classify it as an anxiety patient. Among them, the score of mild anxiety is $50-59$, the score of moderate anxiety is $60-69$, and the score more than 70 is severe anxiety.

\subsubsection{Medical Coping Modes Questionnaire (MCMQ)}

Medical Coping Modes Questionnaire (MCMQ) is used to reflect a patient's coping style and ability in the event of a major illness. The current scale has been partially revised by domestic psychologists on the basis of the original. The scale contains 20 items, which are mainly divided into three dimensions, which are three different ways of coping with "face", "avoidance" and "submission". MCMO 
uses a four-level scoring method to assess the extent of symptoms in each patient. There are 8 items marked with an ${ }^{\star}$ in the entire scale as reverse scoring items, and the scoring standard is reversed from the back to the front. The corresponding items in the three dimensions are cumulatively added to calculate the mean value of the response.

\subsection{Study Method}

\subsubsection{Sampling}

Study objects who met the inclusion criteria were randomly divided into intervention group and comparison group by simple sampling. In the comparison group, only routine treatment was performed, and cognitive behavioral intervention therapy was not performed. The intervention group performed cognitive behavioral intervention therapy while performing routine treatment.

\subsubsection{Intervention Method}

Cognitive behavioral intervention method for "one-to-one" interview on study objects mainly include: 1) Cognitive reconstruction: using rational emotional therapy, adding cognitive factors to behavioral therapy of patients, replacing them with rational thinking methods, to reduce the negative emotions and poor coping styles of patients due to irrational thinking. 2) Behavioral therapy: For patients with excessive anxiety, progressive muscle relaxation therapy is added during the intervention to relieve the patient's nervousness. Progressive muscle relaxation therapy mainly uses the patient's conscious tension and relaxation of muscle groups in various parts of the body, allowing the patient to eliminate tension and slowly achieve a relaxation purpose, thereby adjusting and relieving the patient's stress response and promoting physical and mental harmony. After the intervention object was determined, the object was given a psychological intervention 30 - 40 min per day before surgery, and the intervention period was 1 month. Investigators try their best to be honest in their interventions, avoid using too many technical terms, encourage patients from time to time, and create a pleasant and relaxed conversation atmosphere so that patients can understand and actively cooperate with the study.

\section{Results}

\subsection{Comparison of SAS Scores between the Two Groups before and after Intervention}

The paired sample t-test was used to analyze the SAS scores of the two groups before intervention. There was no significant difference in the SAS scores between the intervention group and the comparison group before intervention $(P>0.05)$. There was a statistically significant difference in SAS scores between the intervention group and the comparison group after intervention $(\mathrm{P}<0.05)$. There was significant difference in the SAS scores between the intervention groups before and after intervention $(\mathrm{P}<0.05)$. There was no significant difference in the SAS scores between the comparison group before and after interven- 
tion $(\mathrm{P}>0.05)$. See Table 2 for details.

\subsection{Comparison of Scores of MCMQ Subscales between the Two Groups before and after Intervention}

The paired sample t-test was used to analyze the scores of three different coping styles of MCMQ in the two groups before intervention. Before intervention, there was no statistically significant difference in the scores of the three coping styles of "face", "avoidance" and "submission" between the intervention group and the comparison group $(\mathrm{P}>0.05)$. After intervention, there was a statistically significant difference in the scores of the three coping styles of "face", "avoidance" and "submission" between the intervention group and the comparison group $(P<0.05)$. There was significant difference in the scores of MCMQ in different coping styles before and after intervention $(\mathrm{P}<0.05)$. There was no significant difference in the scores of the three coping styles of MCMQ between the comparison group before and after the intervention $(\mathrm{P}>0.05)$. See Table 3 for details.

Table 2. Comparison of anxiety scores before and after intervention in two groups of patients $(\bar{x} \pm s)$.

\begin{tabular}{ccccc}
\hline Item & $\begin{array}{c}\text { Intervention Group } \\
(\mathrm{n}=50)\end{array}$ & $\begin{array}{c}\text { Comparison Group } \\
(\mathrm{n}=50)\end{array}$ & $\mathrm{t}$ & $\mathrm{P}$ \\
\hline Anxiety Scores before intervention & $46.32 \pm 8.65$ & $44.33 \pm 7.81$ & 1.207 & 0.115 \\
Anxiety Scores after intervention & $39.19 \pm 7.23$ & $42.18 \pm 7.52$ & 2.027 & 0.023 \\
$\mathrm{t}$ & 4.472 & 1.402 & & \\
$\mathrm{P}$ & 0.000 & 0.082 & \\
\hline
\end{tabular}

Note: $\mathrm{P}<0.05$ was statistically significant.

Table 3. Comparison of the scores of the medical coping styles of the two groups of patients before and after the intervention $(\bar{x} \pm s)$.

\begin{tabular}{|c|c|c|c|c|c|}
\hline \multicolumn{2}{|c|}{ Coping Style } & $\begin{array}{l}\text { Intervention Group } \\
\qquad(\mathrm{n}=50)\end{array}$ & $\begin{array}{c}\text { Comparison Group } \\
(\mathrm{n}=50)\end{array}$ & $\mathrm{t}$ & $\mathrm{P}$ \\
\hline \multirow{4}{*}{ Face } & Before intervention & $18.42 \pm 4.27$ & $19.14 \pm 2.93$ & 0.983 & 0.164 \\
\hline & After intervention & $22.56 \pm 2.55$ & $18.97 \pm 1.96$ & 7.893 & 0.000 \\
\hline & $\mathrm{t}$ & 5.886 & 0.341 & & \\
\hline & $\mathrm{P}$ & 0.000 & 0.367 & & \\
\hline \multirow{4}{*}{ Avoidance } & Before intervention & $15.64 \pm 2.69$ & $16.03 \pm 2.31$ & 0.778 & 0.219 \\
\hline & After intervention & $13.53 \pm 1.78$ & $16.31 \pm 1.93$ & 7.487 & 0.000 \\
\hline & $\mathrm{t}$ & 4.625 & 0.658 & & \\
\hline & $\mathrm{P}$ & 0.000 & 0.256 & & \\
\hline \multirow{4}{*}{ Submission } & Before intervention & $8.69 \pm 2.75$ & $8.58 \pm 2.31$ & 0.217 & 0.415 \\
\hline & After intervention & $7.39 \pm 1.61$ & $9.03 \pm 1.81$ & 4.487 & 0.000 \\
\hline & $\mathrm{t}$ & 2.885 & 1.084 & & \\
\hline & $\mathrm{P}$ & 0.002 & 0.140 & & \\
\hline
\end{tabular}

Note: $\mathrm{P}<0.05$ was statistically significant. 


\section{Discussion}

\subsection{Effect of Cognitive Behavioral Intervention on Anxiety in Breast Cancer Patients}

The results of this study showed that, the anxiety scores of the intervention group and the comparison group before the intervention were not statistically significant. After the cognitive behavior intervention combined with psychology, the anxiety scores of the intervention group and the comparison group were significantly lower than those of the comparison group before intervention, although the anxiety score of the comparison group also decreased, the anxiety score of the intervention group decreased more significantly. Before the intervention, there was no significant difference in anxiety scores between the two groups, but after the intervention, the anxiety difference between the two groups was statistically significant. There was a statistically significant difference in anxiety scores between the intervention group and the intervention group; there was no significant difference between the comparison group and the intervention. The results of the study indicate that cognitive behavioral intervention has a good effect on improving anxiety in breast cancer patients, and it is an effective intervention, this is related to the emotional intervention of breast cancer patients with cognitive behavioral intervention therapy, and the results are basically the same [8] [9].

This study suggests that cognitive behavioral intervention therapy has a positive effect on the psychological state of breast cancer patients. During the test, we gave patients psychological and spiritual care, comfort and support through psychological intervention such as cognitive reconstruction and progressive muscle relaxation, so that patients can understand their negative emotions for surgical treatment and the serious impact of postoperative rehabilitation. Let patients correct their own false perceptions, learn to self-regulate, improve bad coping styles, and be able to recognize their own diseases with a positive and optimistic attitude. The results of this study showed that cognitive behavioral intervention therapy can improve patients' mental state and other symptoms in a certain degree, reduce their negative emotions and reduce their emergency level.

\subsection{Effect of Cognitive Behavioral Intervention on Medical Coping Styles of Breast Cancer Patients}

The results of this study showed that the three ways of medical coping styles in the intervention group and the comparison group of breast cancer patients, "face", "avoidance" and "submission", were the same, and the groups were balanced and comparable. There was no significant difference in the "face", "avoidance" and "submission" of the medical coping style between the intervention group and the comparison group before intervention. After the cognitive behavioral intervention, the "face" coping style of breast cancer patients in the intervention group gradually increased with the advancement of intervention therapy, and the "avoidance" and "submission" coping styles gradually decreased 
with the advancement of intervention therapy. The results showed that with the intervention treatment, cognitive behavioral intervention therapy can effectively improve the medical coping style of breast cancer patients with psychological stress, enabling patients to reduce the coping styles of "avoidance" and "submission" and reduce the degree of "avoidance" and "submission", improve the coping style of "face". This result is similar to the results of domestic and international scholars using cognitive behavioral therapy for medical coping methods of cancer patients [10] [11]. The reason for the analysis is probably due to the lack of disease-related knowledge of the patient itself, and the stress factors such as the fear of the disease, the therapeutic effect, the prognosis, and the like, and the high expectations. In addition to suffering from the pain of the disease itself, the patient has to endure psychological pain, so it is more prone to a weak, self-defeating feeling of powerlessness. As the operation time is closer, the bad stress mode produces negative psychology. The greater the impact, the more patients tend to adopt the coping style of "avoidance" and "submission". After the patient in the intervention group had some psychological intervention, she was able to take the initiative to recognize her own bad coping style, take the initiative to adjust, and adopt a more positive "face" coping style to receive treatment.

In this study, we found that, through the SAS and MCMQ scores in breast cancer patients, positive cognitive behavioral intervention can effectively reduce the stress response of breast cancer patients and improve the medical coping style of patients. Reasonable intervention therapy has a good application value in clinical application and is suitable for application to clinical departments. However, due to the limitation of time and place, this study only conducted intervention tests in a hospital's breast surgery. The sample coverage was too small and the sample size was limited. Because of the subjective factors, the interventions in this study only used behavioral therapy with cognitive reconstruction and progressive muscle relaxation training. Other intervention methods were not involved. Therefore, further study and discussion are still needed. Moreover, the human resources limited by the combination of clinical knowledge and psychological knowledge of hospital medical staff have not yet reached the conditions for comprehensive promotion, so this cognitive behavioral intervention can only be gradually promoted in clinical application.

\section{Funding}

Hainan Natural Science Foundation, Number: 818MS172; Hainan Science and Technology Plan Project: ZDYF2016109; Funded by the National Natural Science Foundation of China, No. 81760255.

\section{Conflicts of Interest}

The authors declare no conflicts of interest regarding the publication of this paper.

\section{References}

[1] Liu, L. and Ren, X.H. (2009) Advances in Intervention of Cognitive Behavioral 
Therapy on Postoperative Psychological Stress in Patients with Breast Cancer. Chinese Journal of Practical Nursing, 25, 74-76.

[2] Yu, Z.G. (2010) Epidemiological Characteristics and Influencing Factors of Breast Cancer in Women in Some Areas of China. National Conference of Endocrinology Surgery, Chinese Medical Association.

[3] Qu, Y.M. and Yang, D.M. (2015) Pay Attention to Stress Psychological Training and Maintain the Physical and Mental Health Development of Middle School Students. Reading and Writing, No. 40, 1.

[4] Zhang, X. (2009) Effects of Psychological Behavior Intervention on Quality of Life, Mood and Coping Style of Cancer Chemotherapy Patients. Central South University, Changsha.

[5] Shakeri, J., Kamangar, M., Ebrahimi, E., Aznab, M. and Shakeriet, H. (2015) Association of Coping Styles with Quality of Life in Cancer Patients. Indian Journal of Palliative Care, 21, 298-304. https://doi.org/10.4103/0973-1075.164890

[6] Wang, Y. (2018) Study on the Effect of Psychological Intervention on Psychological Stress and Cor and IL-2 in Perioperative Breast Cancer Patients. Jilin University, Changchun.

[7] Dukes, C., Thompson, S.B. and Heathcote, D. (2013) Treating PTSD in Cancer Patients. Webmedcentral Com Treating PTSD Patients.

[8] Sunderland, M., Wong, N., Hilvertbruce, Z. and Andreqs, G. (2015) Investigating Trajectories of Change in Psychological Distress amongst Patients with Depression and Generalised Anxiety Disorder Treated with Internet Cognitive Behavioural Therapy. Behaviour Research \& Therapy, 50, 374-380. https://doi.org/10.1016/j.brat.2012.03.005

[9] Hong, S.Y. (2015) Analysis of the Effect of Cognitive Behavior Nursing Intervention on Negative Emotion in Patients with Breast Cancer after Operation. Medical Theory and Practice, No. 24, 3426-3427.

[10] Matthews, E.E., Schmiege, S.J., Cook, P.F., Berger, A.M. and Aloia, M.S. (2012) Adherence to Cognitive Behavioral Therapy for Insomnia (CBTI) among Women Following Primary Breast Cancer Treatment: A Pilot Study. Behavioral Sleep Medicine, 10, 217-229. https://doi.org/10.1080/15402002.2012.666220

[11] Gao, J.H. (2016) Cognitive Behavioral Intervention of the Coping Styles in Patients with Gastrointestinal Cancer Chemotherapy-Problem Based Solving Therapy. Second Military Medical University, Shanghai. 